facilitating substantial improvements in both patient and service outcomes.

\section{HEALTHCARE LEADERSHIP IN MEDICAL SCHOOL: DELIVERING EXTRA-CURRICULAR LEADERSHIP TEACHING AND OPPORTUNITIES THROUGH STUDENT- SOCIETY EVENTS}

${ }^{1} \mathrm{M}$ Shihabul Hassan, ${ }^{1}$ Prisca Singh, ${ }^{2}$ Haroon Shah. ${ }^{1}$ College of Medical and Dental Sciences, University of Birmingham Medical School, UKi ${ }^{2}$ Queen's Hospital Burton, UK

\subsection{6/leader-2021-FMLM.28}

Introduction The experiences of medical students suggest that the teaching on healthcare leadership currently delivered through core curriculum material at medical school is insufficient, and there are few opportunities to engage in leadership activities. Thus, Birmingham Medical Leadership Society (BMLS) sought to gauge students' perceptions on an alternative form of healthcare leadership teaching that would facilitate core curriculum material - extracurricular events on healthcare leadership and management organised by a studentled society.

Methods Renowned leaders in healthcare were invited to speak on various topics related to leadership and management at 12 online events organised by BMLS. 249 participants completed evaluation forms, distributed after each event. Participants were asked to rate the leadership training available at their medical school and the perceived usefulness of the BMLS event they attended, how interesting it was, and how relevant it would be to their careers - on a scale of 1 to 5 , with 5 being most useful/interesting/relevant.

Results Only $27 \%$ of participants agreed or strongly agreed that the leadership teaching or opportunities available at their medical school were sufficient for developing leadership skills for the workplace. $96 \%$ of the 249 participants rated the BMLS event they attended $5 / 5(62 \%)$ or $4 / 5(34 \%)$ in terms of usefulness. $92 \%$ rated the event $5 / 5(62 \%)$ or $4 / 5(31 \%)$ in terms of how interesting it was. $94 \%$ rated the event $5 / 5$ $(66 \%)$ or $4 / 5(28 \%)$ on relevance to future careers.

Conclusion This study demonstrates that the use of the student-society model for promoting healthcare leadership and management is an effective way to incorporate leadership training at medical school without overloading medical curricula. Existing medical leadership societies across the nation are recommended to adopt the scalable, sustainable, and promising student-society model demonstrated, to promote healthcare leadership among students.

\section{HIGH OUTPUT VACCINATION CENTRE FOR OPTIMUM VACCINE DELIVERY}

${ }^{1}$ Reshma Rasheed, 'Babafemi Salako, 'Olugbenga Odutola, ${ }^{2}$ Yathorshan Shanthakumaran. ${ }^{1} B B$ Healthcare Solutions, UK; ${ }^{2}$ University of Varna, Bulgaria

10.1136/leader-2021-FMLM.29

Background The COVID-19 pandemic presented many challenges, one of which was the necessity to provide a large number of COVID-19 vaccines at scale within a short period of time.

Aims and Objectives To use process mapping to ensure maximum efficiency flows of patients through a High Output
COVID vaccination centre during the COVID-19 pandemic and utilize lean methodology and six sigma methods to ensure maximum output within both time and resource constraints in primary care.

Methods Using process mapping and patient flow mapping, we were able to study various permutations of COVID-19 vaccine pathways from booking to vaccine delivery. The service was challenged by practices to deliver the most effective patient vaccine volumes within a given time frame ensuring patients could be streamed through the vaccine centres with maximal efficiency. The high output vaccine model could ensure a maximum uptake of 2500 vaccines in one working day. Following the initial pilots, the process flow mapping enabled the application of lean methodology and six sigma to reduce inefficiency and duplication. The design was implemented across three sites across the Basildon, Billericay and Wickford areas optimising workflow and execution of large volumes of vaccine delivery during the national immunisation programme.

Results The methodology enabled a process flow map that would provide the most clinical and cost effective patient flow through the high output vaccine hub ensuring maximum numbers could be vaccinated in the given time.

Conclusions The QI methodology enabled a cost effective and clinically effective high output vaccine hub to provide up to 2500 vaccines in one day, across three sites in the Basildon and Brentwood, Wickford area.

\section{HOSPITAL 2 HOME: IMPLEMENTATION OF A CLOSED- LOOP DIGITAL PATHWAY TO SUPPORT REHABILITATION IN AN OLDER POPULATION}

1,2Sam Fosker, ${ }^{1,2}$ Kalon Hewage, ${ }^{3}$ Christina Koulouglioti, ${ }^{3}$ Robert Wagner, ${ }^{3}$ Richard Venn, ${ }^{3}$ Luke Hodgson. ${ }^{1}$ Cush Health Limited; ${ }^{2}$ NHS Clinical Entrepreneurs; ${ }^{3}$ University Hospitals Sussex NHS Trust UK

\subsection{6/leader-2021-FMLM.30}

Aims To establish the feasibility of a closed-loop feedback system to remotely monitor and deliver personalised care to patients who have had a fractured Neck of Femur (NoF).

Methods Smartwatches were provided to NoF patients post repair and linked to the Cush Health digital web-app platform allowing visualisation of the data by the patient and their clinical team and access to personalised exercise/support plans. Monthly multidisciplinary meetings attended by the healthcare team reviewed smartwatch data and patient progress to progress rehabilitation. Objective outcomes (ie. smartwatch and quality of life data) was combined with subjective feedback from patients and the clinical team.

Results 3-month data showed positive engagement with technology and a progressive increase in step count data, reduction in average heart rate and reduced length of stay in a 62 89 years old population. Clinical lessons learnt include specific drivers and barriers for older populations; use of support networks can help engagement in those not experienced with technology; closed-loop system can empower patient autonomy and progress rehabilitation whilst an inpatient and help transition to community care. Independent analysis of the pathway showed cost and carbon emission saving benefits by facilitating a remote MDT and patients have responded positively by having the supported messaging via the platform.

Conclusion Our research has shown that a rapid deployment of a digital pathway is feasible and able to deliver better 
tailored rehabilitation to a wide number of patients. Further work needs to be done to establish best methods for engaging older populations and specifically those not confident in use of technology.

Technologies such as this have the potential to support patients from hospital to home, lending themselves to integration into the plans for the Integrated Care System

\section{HOW A JUNIOR DOCTOR BODY CAN HELP COMMUNICATION, AND IMPROVE MORALE IN A LARGE TEACHING HOSPITAL TRUST}

Michael Ewens, Stuart Haines, Laura Brown, Louise Buchanan, Dipesh Odedra. Leeds Teaching Hospitals NHS Trust

10.1136/leader-2021-FMLM.31

Leeds Teaching Hospitals NHS Trust (LTHT) has one of the largest cohorts of Junior Doctors in England, employing approximately 1,000 at any one time. Rotations occur every 4-12 months, before Juniors move to neighbouring trusts for further training.

LTHT's Chief Registrar chairs the Junior Doctor Body (JDB), a group of Junior Doctors, that meet virtually, monthly, representing each Clinical Service Unit and grade of Junior (Foundation Year to Registrar). The aim of the JDB is to increase communication between LTHT's Executive Team and Junior Doctors, who quite often do not feel a sense of belonging with their employer due to the short nature of employment.

In 2020/21, the JDB increased from 12 members, to 32, through a targeted communications campaign by the Chief Registrar. The inclusion of our Professional Support Unit helped highlight health and wellbeing resources for Junior Doctors within LTHT. This safe space allowed discussion of issues important to Junior Doctors, including: adequate rest facilities during the COVID-19 pandemic, refreshments, annual leave and impact of COVID on training opportunities and progression.

Each month, JDB reps were able to highlight any issues within their department that were not solved locally. Opportunities were cascaded to help juniors continue to meet training needs, including: quality improvement training and participation in collaboratives, COVID-19 vaccination research, and the chance to create educational resources in the correct use of personal protective equipment.

Executive Team members delivered talks on inclusion and diversity, patient safety and their career journeys; keen to ask for feedback to further make LTHT the very best place to train. Issues were able to be solved immediately within JDB meetings, helping Junior Doctors feel appreciated and respected; boosting morale during what has been a difficult year. We encourage all trusts to replicate the JDB model to boost morale nationally.

\section{IMPLEMENTATION OF AN ONLINE MICROBIOLOGY REFERRAL PATHWAY IN A HOSPITAL TRUST - A QUALITY IMPROVEMENT REPORT}

${ }^{1}$ Kim Pramanik* ${ }^{2}$ Sandra Long. 'University Hospitals Leicester, Infirmary Square, Leicester, UK, LE1 5WW; ${ }^{2}$ Royal Blackburn Hospital, Haslingden Road, Blackburn, UK, BB2 3HH
Aims Appropriate diagnostic and antimicrobial stewardship relies on national and local data on antimicrobial resistance patterns unique to every region. Adaptive problems such as variability in the quality of information provided for antimicrobial guidance, inefficiency of a phoned referral system and reduced compliance with Royal College of Pathologists key performance indicator 6.3 of response to urgent referrals within one hour mandated a microbiologist-led change of referral method and content to improve assessment and management of a suspected infectious disease.

Methods A measurement plan comprising parameters quantifying the problems associated with phoned referrals was used over two plan do study act (PDSA) cycles. Approximately 25 patients were sampled randomly for urgent and non-urgent referrals during 2020 and 2021 using online consultation records. Interventions for this project were an online nonurgent referral pathway using existing trust-approved software rolled out for over four million patients, and online communications disseminated trust-wide to all clinicians for awareness and engagement. Feedback was sought in a third PDSA cycle using a survey via trust email, enabling sampling of clinician impressions.

Results Compliance improved in all parameters, repeat calls were prevented on re-assessment, and non-urgent referral information quality increased to $100 \%$ from 24\%. Survey results $(n=19)$ revealed that $89 \%$ preferred the online referral method, though increased awareness of the pathway was needed.

Conclusions Continued use would improve efficiency and transparency of communication, documentation and enable data collection for governance. Implementation of further PDSA cycles could promote sustainability and eventually reduce the risk of antimicrobial resistance. Future plans are in place to expand the clinician base to general practitioners within the region.

*Presenter

\section{IMPLEMENTING NEW TECHNOLOGY IN THE OUTPATIENT SETTING: CHALLENGES AND OUTCOMES IN BLADDER DIAGNOSTIC ENDOSCOPY}

${ }^{1}$ Lara Ratcliffe, ${ }^{2}$ Brian Birch. ' University of Southampton, School of Medicine, Southampton, UK: ${ }^{2}$ University Hospital Southampton, Southampton, UK

\subsection{6/leader-2021-FMLM.33}

Aims To implement the use of an endosheath-covered cystoscope - a disposable sheath that fits over the cystoscope

Perform a service evaluation of performance of the new sheathed scope

To maintain safety and efficiency in the department

Become a leading centre for improvements in flexible cystoscopy techniques

Methods Participants undergoing flexible cystoscopy at Lymington hospital, UK between January 2018 to April 2020 participated in this service implementation of endosheath covered flexible cystoscopy. This received high levels of staff and participant engagement, quickly becoming part of the unit culture.

Participants completed 2 surveys, the first immediately post procedure; discomfort was rated on a 10 point visual analogue scale $(1=$ no discomfort, $10=$ extreme discomfort). The second survey completed $\sim 2-4$ weeks later, development of 